\title{
The Effects of Trauma-Focused Research on Pregnant Female Participants
}

KAMI L. SchWERDTFEgeR

Oklahoma State University at Stillwater

Briana S. Nelson Goff

Kansas State University at Manhattan

\begin{abstract}
RECENT EMPHASIS ON THE ETHICAL CONDUCT of researchers has resulted in a growing body of literature exploring the impact of trauma-focused research on participants. To date, pregnant women have not been widely included in trauma-focused research, possibly because they are considered a vulnerable population in research. The current research investigated how 41 expectant mothers responded to participation in a trauma-focused study. Overall, the results of this study suggest that trauma-focused research is well tolerated by pregnant women. Specific findings indicated that pregnant women considered more traumatized perceived greater benefits from participation. Best practices for conducting ethical trauma-focused research with pregnant women are provided. As well, recommendations for future research are outlined in an effort to further extend the ethical understanding of the benefits and costs of trauma-focused research with potentially vulnerable populations.
\end{abstract}

KEY WORDS: trauma-focused research, research subjects, pregnancy, women, ethics, risk-benefit analysis

Received: September 18, 2007; revised: January 4, 2008

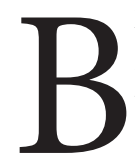
ECAUSE TRAUMA-FOCUSED RESEARCH OFTEN involves asking participants about difficult or traumatic experiences, the issue of participant risk is of critical importance. Until recently, little evidence on trauma-focused research was available to assist in the appropriate assessment of the risk-to-benefit ratio facing potentially vulnerable participants.

Recent studies focusing on the effects of traumafocused research participation have generally found participation to be well tolerated (Newman, Risch, \&
Kassam-Adams, 2006). In general, the literature suggests that although trauma-based research may produce intense emotions, it is not re-traumatizing nor does it cause harm to participants. Studies involving a variety of trauma survivors found that participation in the research was not overwhelming or distressing and was generally an experience that participants would be willing to repeat (Carlson et al., 2003; Griffin, Resick, Waldrop, \& Mechanic, 2003; Kassam-Adams \& Newman, 2002; Newman, Walker, \& Gefland, 1999; Newman, Willard, Sinclair, \& Kaloupek, 2001; Parslow, Jorm, O’Toole, Marshall, \& Grayson, 2000; Walker, Newman, Koss, \& Bernstein, 1997).

\section{PREGNANT FEMALES AS PARTICIPANTS}

IN HUMAN-SUBJECTS RESEARCH

According to Newman et al. (2006), certain populations of trauma survivors may require additional attention when considering research ethics or safety concerns. Absent from their description are pregnant females. Despite the significant gains that have been made in developing an empirical basis for conducting ethical trauma-focused research with various populations, no research has specifically investigated the impact of participation in trauma-focused research on pregnant women. When a pregnant female agrees to take part in a research study, she is considered to be providing informed consent for two people: herself and her fetus.

In general, the inclusion of pregnant women in human-subject research has required special ethical considerations that assure protection for both the expectant mother and the fetus. Under federal regulations, special conditions and additional safeguards must be considered when pregnant women are recruited for research participation. As stated in these guidelines, research may be conducted with pregnant women only if both the mother and fetus are placed at minimal risk (US National Commission for the Protection of Human Subjects of Biomedical and Behavioral Research, 1979). The regulatory issues outlined by 45 CFR 46 Subpart B (US National Commission for the Protection of Human Subjects of Biomedical and Behavioral Research, 1979) specify the special protections for all pregnant women

Journal of Empirical Research on Human Research Ethics, PP. 59-67. PRINT ISSN 1556-2646, ONLINE ISSN 1556-2654. () 2008 BY JOAN SIEBER. ALL RIGHTS RESERVED. PLEASE DIRECT ALL REQUESTS FOR PERMISSIONS TO PHOTOCOPY OR REPRODUCE ARTICLE CONTENT THROUGH THE UNIVERSITY OF CALIFORNIA PRESS'S RIGHTS AND PERMISSIONS WEBSITE, HTTP://WWW.UCPRESSJOURNALS.COM/REPRINTINFO.ASP. DOI: $10.1525 /$ jer.2008.3.1.59 
and fetuses. Although pregnant females are generally not excluded from studies that include surveys, interviews, and other forms of non-invasive data collection, federal regulations classify all pregnant women as vulnerable, even though women are seldom vulnerable because of pregnancy alone.

There are several reasons why pregnant females may be considered at-risk or more vulnerable to research participation. Some might argue that they are biologically vulnerable during pregnancy and might be more sensitive or vulnerable because of their biological/hormonal changes. Others might consider them to be more vulnerable because of possible current trauma risks and that participating in research could put them at increased risk for pregnancy-related traumas (e.g., miscarriage, interpersonal violence during pregnancy). Or, pregnant females could possibly be more vulnerable because of the emotional changes that occur; reactions due to questions about previous trauma experiences may be more prominent during pregnancy, thus putting them at increased emotional risk.

According to Kipnis (2005), because vulnerability is relative to context, having a vulnerable category that classifies or stigmatizes a group of people (such as pregnant women) as vulnerable is not warranted. It is more adequate to consider that a pregnant woman might at certain times and under certain circumstances be medically vulnerable to losing her fetus. Therefore, special protections geared toward regulating biomedical research protocols, which may involve heightened risk of fetal loss, may not adequately pertain to the minimal risks generally associated with social and behavioral research (Kipnis, 2005). Despite the distinct differences in risk, research must be conducted to understand whether vulnerabilities exist for pregnant women and their fetuses who participate in social and behavioral research, specifically trauma-focused research.

Although there have been no studies assessing the impact of trauma-focused research participation on pregnant women, there is significant research indicating that trauma research participation is well tolerated among nonpregnant populations. This may suggest a similar risk-to-benefit ratio among pregnant female participants. However, more information is needed to assist researchers and IRBs in making ethical decisions and identifying legitimate concerns regarding research protocols with pregnant women.

The current study examined data drawn from an exploratory trauma study assessing the relationship between experienced trauma and attachment history of expectant mothers and the subsequent development of prenatal attachment to their unborn children (Schwerdtfeger \& Nelson Goff, 2007). As part of this study, pregnant women completed a self-report questionnaire to assess their responses to research participation. This questionnaire was added to the research protocol due to concerns raised during the IRB review process. The IRB raised questions about potential risks the trauma-focused research could present to the pregnant female participants, particularly because of the focus on previous trauma exposure and current trauma symptoms. Thus, the current project was designed to shed light on the reactions and potential risks of pregnant women participating in traumarelated research. As suggested by previous studies, it was hypothesized that the pregnant female participants would report minimal distress and positive appraisals of research participation. However, in order to understand possible trauma-specific factors that might be associated with distress reactions in the research participants (Newman et al., 2006), analyses were conducted on two specific variables to determine the impact on participants' reactions: lifetime trauma exposure and trauma symptoms.

\section{Method}

\section{PARTICIPANTS}

The sample for the current study was assembled over a 3 -month period. Research participants were pregnant women seeking prenatal health care at a county health department in the Midwest. This clinic served primarily lower socioeconomic status individuals and families. Criteria for inclusion were that the expectant mother be at least 18 years of age and in the second or third trimester of pregnancy.

\section{SELECTION OF PARTICIPANTS}

In order to obtain the desired sample of participants, individuals meeting the specified criteria were introduced to the nature of the study and invited to participate by staff members of the county health department. If the participant agreed, the researcher provided more information about the study. Participants were informed that the study included a number of questions about their past life experiences, including previous difficult or traumatic events. In addition, information about confidentiality and participants' right to refuse participation at any time were discussed. Participants were informed of a monetary incentive for their participation (a \$10 gift card to a local discount store). After being introduced to the study and agreeing to participate, participants were asked to review and sign the informed consent. 


\section{ENHANCING PARTICIPANT PROTECTION}

Based on the IRB review, several procedures were included as additional assurances in monitoring and limiting participant distress during data collection. First, all participants were given the option of completing the study measures in a paper-and-pencil format, in which the participant read the questions and marked their responses, or in an interview format, in which the researcher read the questions aloud and recorded the participants' responses. Most participants $(n=38$, $92.7 \%$ ) opted to complete the study measures in the interview format, with $7.3 \%(n=3)$ completing the measures individually in the paper-and-pencil format.

In the middle of each data-collection session, the researcher verbally and behaviorally assessed participants' levels of distress and comfort and offered the participant a five-minute break. Before beginning the second half of the study, participants were asked to review and sign an additional informed consent form for continuation in the study. Throughout the data collection, the researcher took notes, which included information on participants' distress and any additional information participants provided while responding to the quantitative measures. Upon completing the study measures, participants were provided with a copy of their signed informed consent, an informative handout on common symptoms related to traumatic experiences, a debriefing statement, and the monetary incentive.

\section{Measures}

REACTIONS TO TRAUMA RESEARCH PARTICIPATION

The Reactions to Research Participation Questionnaire Revised (RRPQR; Newman et al., 2001) was added in response to initial concerns of the university IRB and was administered to quantify participants' response and perception of the risks and benefits of participating in trauma-focused research. (For further discussion of the RRPQR and access to the instrument, see http://www. personal.utulsa.edu/ elana-newman/.) By assessing the impact of research on trauma survivors, the RRPQR is designed to provide researchers and IRB members with important information regarding the cost-to-benefit ratio associated with trauma-focused research.

The RRPQR consists of 24 items assessing participants' experience of study participation, reasons for participation, and perceived costs and benefits of participation. The measure contains specific items addressing the experiences of participation, including perceived coercion or undue influence by the investigators and perceptions of the researcher's respect for the individual, as well as participants' perceptions of personal gain, meaningfulness, and discomfort associated with research participation. Individual items are rated on a 5point Likert scale ranging from 1 (strongly disagree) to 5 (strongly agree). Negatively worded items are reverse scored. Higher scores indicate more favorable reactions to research participation. The maximum total score on the RRPQR is 115 , with a minimum score of 23 . In addition to the individual items and total scores, the RRPQR consists of five subscales: Participation, Personal Benefits, Emotional Reactions, Perceived Drawbacks, and Global Evaluation Factor. Reliability of the Reactions to Research Participation Questionnaire (RRPQ; Newman et al., 2001), the measure from which the RRPQR was derived, is adequate, with an alpha of .83. For the current study, reliability of the RRPQR was also found to be adequate, with an alpha of $.79(N=41)$.

LIFETIME TRAUMA EXPOSURE

Lifetime trauma exposure was measured using the Traumatic Events Questionnaire (TEQ; Vrana \& Lauterbach, 1994). The TEQ is a frequently used research measure assessing participants' experiences with nine specific categories of traumatic events (accidents, natural disasters, crime, child abuse, rape, adult abusive experiences, witnessing the death/mutilation of someone, being in a dangerous/life threatening situation, receiving news of the unexpected or sudden death of a loved one) reported in the DSM-III-R and the empirical literature as having the potential to elicit posttraumatic stress symptoms.

The TEQ contains a list of 16 traumatic events. Participants indicated whether or not they had experienced the event by circling "Yes" or "No" next to the event listed. In addition, two residual categories are included, allowing respondents to report any other events or personal experiences not previously listed, but considered by the participant as traumatic. For the current study, the primary focus was on the presence/ absence of various traumatic events rather than the severity of such experiences. Therefore, a trauma exposure score ranging from 0 to 18 was obtained by summing the number of events the participant reported experiencing. For the current study, reliability of the TEQ was adequate with an alpha of .66 .

\section{TRAUMA SYMPTOMS}

The Trauma Symptom Checklist-40 (TSC-40; Briere, 1996) is a 40-item self-report research measure assessing symptomatology of adults resulting from childhood and/or adult traumatic experiences. The instrument consisted of six subscales representing six types of trauma related symptoms: Anxiety, Depression, Dissociation, 
Sexual Abuse Trauma Index, Sexual Problems, and Sleep Disturbance. Sample symptoms from the subscales included tension, sadness, low sex drive, feeling that things are unreal and insomnia. Responses to each symptom item were rated on a 4-point Likert scale, ranging from 0 (never) to 3 (often), indicating how much the occurrence bothered the participant over the previous two months, not related to pregnancy. Total scores on the TSC-40 range from 0 to 120 . Reliability of the TSC-40 was adequate with alphas for the full scale averaging .89 to .91 and subscale alphas ranging from .66 to .77 (Briere, 1996). For the current study, reliability was found to be adequate, with an alpha of $.89(N=41)$.

\section{Results}

PARTICIPANT CHARACTERISTICS

Of 51 potential participants recruited, a total volunteer sample of 41 pregnant females completed the study (response rate $=80 \%), 9$ declined participation in the study, and one was not eligible to participate due to not meeting the minimum age requirement. Typical reasons for refusal included time and schedule restraints. Of the 41 participants, the mean age was 22.9 years $(S D=3.77$, Range $=18-33$ years). Table 1 provides demographic data for the 41 participants.

No participants chose to end the research protocol before completion. In reporting the most important reason for deciding to participate in the current study, the majority of participants reported, "I was curious" $(56.1 \%, n=23)$, with $31.7 \%(n=13)$ reporting "to help others," 9.8\% $(n=4)$ reporting "to help myself," and one participant reporting "other," stating "why not, [I'm] doing nothing else."

Participants ranged from 13 to 41 weeks in their pregnancies. Nineteen (46.3\%) participants reported that it was their first pregnancy. Most of the participants reported that the current pregnancy was unplanned $(n=35,85.4 \%)$. Independent sample $t$ tests were run to compare women on lifetime trauma exposure, trauma symptoms, and reaction to research on the basis of the current pregnancy being planned or unplanned; however, no significant differences were found.

Of the 41 participants, $87.8 \%$ (36 of 41 ) reported a previous trauma history, and the mean number of lifetime traumas experienced was $3.02(S D=2.36)$. Of the 36 participants who reported a previous trauma history, 23 (56.1\%) indicated having experienced interpersonal trauma, including childhood sexual abuse $(n=13$, $31.7 \%)$, childhood physical abuse $(n=10,24.4 \%)$, adult sexual victimization $(n=6,14.6 \%)$, adult domestic
TABLE 1. Characteristics of Participants $(N=41)$.

\begin{tabular}{|c|c|c|}
\hline & $N$ & $\%$ \\
\hline \multicolumn{3}{|l|}{ Ethnicity } \\
\hline Caucasian & 22 & 53.7 \\
\hline African American & 10 & 24.4 \\
\hline Mexican American & 4 & 9.8 \\
\hline American Indian & 2 & 4.9 \\
\hline Asian/Pacific Islander & 1 & 2.4 \\
\hline "Other" & 2 & 4.9 \\
\hline \multicolumn{3}{|l|}{ Relationship Status } \\
\hline Dating/Single & 20 & 48.8 \\
\hline Married & 9 & 22.0 \\
\hline Living w/significant other & 9 & 22.0 \\
\hline Divorced & 3 & 7.3 \\
\hline \multicolumn{3}{|l|}{ Religion } \\
\hline Protestant/Christian & 17 & 41.5 \\
\hline No religious affiliation & 10 & 24.4 \\
\hline Catholic & 7 & 17.1 \\
\hline Non-denomination & 6 & 14.6 \\
\hline Muslim & 1 & 2.4 \\
\hline \multicolumn{3}{|l|}{ Annual Gross Family Income } \\
\hline Below $\$ 9,999$ & 19 & 46.3 \\
\hline$\$ 10,000$ to 19,999 & 12 & 29.3 \\
\hline$\$ 20,000$ to 29,000 & 7 & 17.1 \\
\hline$\$ 30,000$ to 39,000 & 1 & 2.4 \\
\hline$\$ 40,000$ to 49,000 & 1 & 2.4 \\
\hline$\$ 50,000$ to 59,000 & 1 & 2.4 \\
\hline \multicolumn{3}{|l|}{ Education } \\
\hline Some college & 17 & 41.5 \\
\hline High school diploma & 15 & 36.6 \\
\hline Some high school & 8 & 19.5 \\
\hline Completed grade school & 1 & 2.4 \\
\hline \multicolumn{3}{|l|}{ Employment } \\
\hline Unemployed (not disabled) & 15 & 36.6 \\
\hline Employed full time & 11 & 26.8 \\
\hline Employed part time & 7 & 17.1 \\
\hline Full time student & 5 & 12.2 \\
\hline
\end{tabular}

violence $(n=16,39 \%)$, or victimization in a violent crime $(n=9,22 \%)$. Non-interpersonal traumas were reported by $18(43.9 \%)$ participants, which included being in or witnessing a serious accident $(n=11$, $26.8 \%)$; experiencing a natural disaster $(n=8,19.5 \%)$; witnessing the serious injury, mutilation or violent death of someone ( $n=11,26.8 \%)$; receiving news of the unexpected death of someone close to them $(n=24,58.5 \%)$; and being in serious danger of losing one's life or being seriously injured $(n=8,19.5 \%)$. Eight participants reported having experienced a traumatic event that was not listed on the TEQ (e.g., unexpected loss of a sibling or parent, death or torture of a pet/animal, parent incarceration). No participants reported experiencing military related traumas. Descriptive statistics and reliability 
TABLE 2. Descriptive Statistics of Study Measures and Subscales $(n=36)$.

\begin{tabular}{lcc}
\hline & $M(S D)$ & Range \\
\hline TEQ & $3.44(2.21)$ & $1-9$ \\
TSC-40 & $22.06(14.63)$ & $2-59$ \\
$\quad$ Dissociation & $3.50(2.94)$ & $0-11$ \\
$\quad$ Anxiety & $3.72(2.96)$ & $0-11$ \\
$\quad$ Depression & $5.67(4.36)$ & $0-15$ \\
$\quad$ Sexual Abuse & $2.92(3.09)$ & $0-12$ \\
$\quad$ Sleep Disturbances & $6.47(4.46)$ & $0-16$ \\
$\quad$ Sex Problems & $2.28(3.28)$ & $0-13$ \\
RRPQR & $99.86(7.53)$ & $85-114$ \\
\hline
\end{tabular}

of the measures for these 36 participants are presented in Table 2.

TRAUMA HISTORY AND PARTICIPANT REACTIONS

The mean TSC-40 score among the 36 participants with self-reported trauma histories was 22.06 ( $S D=14.63$, possible score from 0 to 120 ), indicating that they were not experiencing significant distress due to trauma related symptoms. Additionally, scores on the RRPQR $(M=99.86, S D=7.53$, possible score from 24 to 115$)$ suggest that the 36 pregnant female participants with previous trauma histories indicated favorable reactions participating in the current trauma-focused research. Responses on individual RRPQR items indicated that all women participating in the current study felt that they were treated with respect and dignity, that they could stop participation at any time, and that they understood the consent form. Descriptive statistics for the 24 items of the RRPQR are presented in Table 3.

Although all participants reported a generally positive reaction to research participation, independent sample $t$ tests were used to compare the 36 women on measures of lifetime trauma exposure and trauma symptoms based on the self report of two potential costs (emotionality and discomfort) and two potential benefits (insight and meaningfulness) associated with participation. Utilizing procedures outlined by Newman et al. (1999), participants were categorized as indicating a positive (4 or 5) or negative (1 or 2 ) reaction based on their responses to four individual RRPQR items: (1) no unexpected emotionality or unexpected emotionality (Item \#3: The research raised emotional issues for me that I had not expected), (2) insight gained or no insight gained (Item \#4: I gained insight about my experiences through research participation), (3) comfort participating or discomfort participating (Item \#5: The research made me think about things I didn't want to think about), and (4) meaningful or not meaningful (Item \#7: I found

TABLE 3. Descriptive Statistics of RRPQR Items $(n=36)$.

\begin{tabular}{lll}
\hline \multicolumn{1}{c}{ Item } & \multicolumn{1}{c}{$M(S D)$} & Range \\
\hline 1. Gained something positive from participating & $3.81(0.67)$ & $3-5$ \\
2. Would participate in the study again & $4.28(0.66)$ & $3-5$ \\
3. Raised unexpected emotional issues* & $3.86(1.22)$ & $1-5$ \\
4. Gained insight through participation & $3.89(0.93)$ & $1-5$ \\
5. Thought about things didn't want to think about* & $4.11(1.12)$ & $2-5$ \\
6. Found questions too personal* & $4.78(0.48)$ & $3-5$ \\
7. Found participating personally meaningful & $3.78(0.90)$ & $1-5$ \\
8. Believe study's results will be useful & $4.22(0.76)$ & $1-5$ \\
9. Trust replies will be kept private & $4.58(0.55)$ & $3-5$ \\
10. Experienced intense emotions* & $4.28(1.09)$ & $2-5$ \\
11. Research is for a good cause & $4.50(0.56)$ & $3-5$ \\
12. Treated with respect and dignity & $4.81(0.40)$ & $4-5$ \\
13. Participating personally beneficial & $3.94(0.79)$ & $2-5$ \\
14. Glad to be asked to participate & $4.31(0.67)$ & $3-5$ \\
15. Liked contributing to science & $4.22(0.64)$ & $3-5$ \\
16. Emotional during research session* & $4.42(0.91)$ & $2-5$ \\
17. Felt could stop participating at any time & $4.64(0.49)$ & $4-5$ \\
18. Found participating boring* & $4.56(0.65)$ & $3-5$ \\
19. Study procedures took too long* & $4.67(0.53)$ & $3-5$ \\
20. Participating was inconvenient* & $4.72(0.51)$ & $3-5$ \\
21. Participation was choice freely made & $4.69(0.52)$ & $3-5$ \\
22. Still would agree to participate & $4.58(0.65)$ & $2-5$ \\
23. Understood the consent form & $3.72(0.45)$ & $4-5$ \\
\end{tabular}

*Reverse scored. 
TABLE 4. Comparison of Participants With and Without Emotionality, Insight, Comfort, and Meaningfulness on Measures of Lifetime Trauma Exposure and Trauma Symptoms $(n=36)$.

\begin{tabular}{|c|c|c|c|c|c|}
\hline & $M(S D)$ & $M(S D)$ & $d f$ & $t$ & $d$ \\
\hline & $\begin{array}{l}\text { Unexpected Emotionality } \\
\qquad(n=24)\end{array}$ & $\begin{array}{l}\text { No Unexpected Emotionality } \\
\qquad(n=7)\end{array}$ & & & \\
\hline TEQ & $3.54(2.06)$ & $2.71(2.17)$ & 29 & -0.90 & 0.40 \\
\hline \multirow[t]{2}{*}{ TSC-40 } & $22.83(13.47)$ & $17.00(13.13)$ & 29 & -1.01 & 0.09 \\
\hline & $\begin{array}{l}\text { Insight Gained } \\
\quad(n=17)\end{array}$ & $\begin{array}{l}\text { No Insight Gained } \\
\qquad(n=4)\end{array}$ & & & \\
\hline TEQ & $2.71(1.76)$ & $5.00(1.83)$ & 19 & 2.33 & -0.32 \\
\hline \multirow[t]{2}{*}{ TSC-40 } & $17.71(11.66)$ & $33.75(15.88)$ & 19 & 2.32 & -0.34 \\
\hline & $\begin{array}{l}\text { Comfort Participating } \\
\qquad(n=26)\end{array}$ & $\begin{array}{l}\text { Discomfort Participating } \\
\qquad(n=5)\end{array}$ & & & \\
\hline TEQ & $3.50(2.25)$ & $4.20(2.77)$ & 29 & 0.62 & -0.06 \\
\hline \multirow[t]{2}{*}{ TSC-40 } & $19.08(12.80)$ & $42.20(10.23)$ & 29 & 3.79 & -0.36 \\
\hline & $\begin{array}{l}\text { Meaningful } \\
\quad(n=26)\end{array}$ & $\begin{array}{l}\text { Not Meaningful } \\
\qquad(n=3)\end{array}$ & & & \\
\hline TEQ & $3.62(2.38)$ & $2.33(0.58)$ & 27 & $-0.91^{*}$ & 0.56 \\
\hline TSC-40 & $22.00(16.02)$ & $19.00(2.00)$ & 27 & $-0.32 *$ & 0.04 \\
\hline
\end{tabular}

${ }^{*} p<.05$.

participating in this study personally meaningful). Participants indicating neutral responses (3) were not included in the analysis for that item. In addition to adequately reflecting the potential costs and benefits associated with trauma-focused research, these four variables were chosen for further analysis as they represent the only four RRPQ-R items for which at least 5\% $(n=36)$ of the women reported negative reactions.

While no differences in lifetime trauma exposure or trauma symptoms were found when comparing pregnant women based on their report of emotionality, insight, or discomfort, significant differences were found based on reports of potential benefits (meaningfulness). Pregnant women indicating that research participation was personally meaningful reported experiencing a significantly greater number of traumatic events and higher trauma symptoms than pregnant women who indicated that participating was not personally meaningful. The data comparing participants' self-report of emotionality, insight, comfort, and meaningfulness on measures of lifetime trauma exposure and trauma symptoms are presented in Table 4.

\section{Discussion}

The goal of the current study was to identify the primary reactions to trauma-focused research participation as reported among pregnant female participants. The results of the current study found that participants generally reported limited negative effects from participating in trauma-focused research. A majority of participants indicated experiencing personal benefits, including insight and meaning as a direct result of research participation. These findings support the claim that pregnant women participating in trauma-focused research are at a low risk of distress or harm. Moreover, these findings suggest that when special attention and additional ethical assurances are employed in trauma-focused research protocol with pregnant female trauma survivors, the benefits of participation in trauma-focused research may outweigh the potential costs.

However, these results must be interpreted with caution. It should be noted that while all 36 participants included in the study had experienced some type of trauma in their past and several participants had experienced multiple traumatic events, the level of current trauma symptoms was relatively low, with a mean of 22.06. Therefore, the pregnant female participants in the current study were not experiencing severe or elevated trauma-related symptoms compared to other samples of female trauma survivors. In a study among childhood sexual abuse survivors, Gold and Cardena (1998) reported an average score of 54.42 on the TSC-40. Gold, Milan, Mayall, and Johnson (1994) reported higher TSC-40 averages in their study of 654 female undergraduate students, with average scores of 70.4 for participants who reported a history of child sexual abuse, 73.5 among participants with a reported history of adult sexual assault, and 77.4 for participants who 
reported a history of both childhood sexual abuse and adult sexual assault. It is possible that a sample exhibiting higher trauma symptoms might report more negative effects from participation in trauma-focused research particularly those with severe PTSD or similar psychiatric symptomatology (Loveland Cook et al., 2004; Seng et al., 2004).

The differences found between pregnant women participants who indicated that research participation was personally meaningful and participants who indicated that participation was not personally meaningful suggests that participants with greater lifetime trauma exposure and higher trauma symptoms may perceive more benefits associated with trauma-focused research participation. In an article highlighting the ethical issues in trauma research, Newman, Kaloupek, Keane, and Folstein (1997) offered that some trauma survivors experience a sense of empowerment when they are able to utilize their own negative experiences to benefit others. The current findings provide support for this claim and suggest that trauma-focused research participation may serve as a meaningful opportunity for trauma survivors to assist others. However, these statistically significant results within the current study should be interpreted with caution due to the small effect size (Cohen, 1969; 1992). The current findings should be interpreted giving special consideration to the particular practices that were included as additional assurances in enhancing participant protection in the current research, which are further discussed and described below.

\section{Best Practices}

In order to increase the protection of participants and reduce potential risk in trauma-focused research, we implemented a two-part data collection procedure separated by a break and an expanded debriefing process that included qualitative questions about the impact of the research on the participants. These components are suggested to provide additional assurances to participants. Also, increased oversight and supervision throughout the data collection process can be beneficial for both participants and researchers. If a participant experiences "greater than minimal distress" or asks not to continue, a clinician or other qualified professional may be brought in to meet conjointly with the researcher and the participant to identify an individual plan for follow-up with the participant. It should be emphasized that in the data collection process, the data collection should involve interaction and the development of a positive rapport with each of the participants, from the initial meeting, rather than the participants simply completing the data collection procedures in isolation.

As mentioned previously, when given the option, most $(92.7 \%)$ of the pregnant women participating in the current study choose to complete the research protocol in a face-to-face interview format, as opposed to the individual paper-and-pencil format also offered. These findings offer possible insight into enhancing the likelihood of trauma survivors' participation in traumafocused research. Providing potential participants with the option to be read questions in a face-to-face interview format may provide a more supportive interpersonal environment for trauma-focused research, potentially resulting in higher response rates and more positive participant reactions to trauma-focused research participation with populations commonly regarded as vulnerable.

\section{Research Agenda}

The current study is unique in that it is one of the first studies to focus on the impact of trauma exposure and symptoms on expectant mothers' reactions to traumafocused research participation. The ethnic diversity of the sample is an important strength, however, the small sample size limits power of the current study and therefore the ability to generalize the findings. The sample was non-random and based only on pregnant women who obtained prenatal medical care at the health department and agreed to participate. However, it also should be noted that the participant sample was not a "typical" sample of pregnant females, as they were young, reported low socioeconomic status, low education levels, and were exposed to multiple previous traumatic experiences. By gaining similar information from a larger, more varied sample, further understanding of how trauma related research participation impacts expectant mothers may be attained. Further, while informative, this sample did not include a control group of nontraumatized pregnant women, or a control group of nonpregnant women who had or had not been traumatized. The addition of those groups would have offered the possibility of more powerful conclusions about the effects of prior trauma and pregnancy.

Additionally, the design of the study used retrospective evaluations of traumatic experiences that included a limited focus on the presence/absence of various prior traumatic events, rather than the severity of such experiences. Future research utilizing longitudinal designs may provide further understanding of the longer lasting costs or benefits associated with research participation. Future investigations including additional assessments 
of the chronicity and recency of trauma experiences would be useful to determine whether potential harm from research is greater among pregnant female participants with reportedly more severe or more recent trauma experiences. To our knowledge, these are new areas of research that require further study with pregnant female participants.

\section{Educational Implications}

While the findings of the current study suggest that pregnant females report a high benefit-to-cost ratio following trauma-focused research participation, we believe that this salutary outcome is due not simply to being asked questions about trauma, but to the creation of a context in which this experience is likely to result in insight and feelings of well-being. In addition to the importance of specific components such as the readability and comprehensive qualities of informed consent documents and debriefing statements (Boothroyd \& Best, 2003; Newman et al., 1999; 2006), special attention should be given to teaching undergraduate and graduate research students as well as investigators about the additional ethical considerations involved in conducting trauma-focused research, particularly when working with vulnerable populations.

The current study can serve as a model for researchers and IRBs examining the ethical considerations regarding cost-to-benefit ratios in trauma-focused research involving pregnant women and other populations commonly regarded as potentially vulnerable. It remains imperative that investigators and IRBs consider the implications of trauma-focused research participation and take the appropriate steps to assure that a safe and ethical research environment exists. However, as with all research involving human subjects, trauma-focused researchers and IRBs must also carefully consider the ethics of not conducting research and the potential of proposed research to produce the information needed to guide clinical treatment and service protocol with specific trauma exposed populations.

While specific attention should always be given to respecting the rights of and providing maximum protection for vulnerable populations, it may be stigmatizing to assume that trauma survivors are too impaired to make informed decisions regarding trauma-focused research participation. As Newman et al. (2006) stated, "Providing the opportunity to participate in trauma research, along with appropriate information regarding costs and benefits, may empower trauma survivors by promoting autonomy" (p. 42). Investigators and ethical review boards should be aware of actual risks and benefits of trauma-focused research to assist them in making these difficult methodological and ethical decisions, as they continue to advance the trauma field through ethical scientific inquiry.

\section{Acknowledgment}

The authors would like to acknowledge Dr. Steve Harris and Chris Peters whose suggestions and comments on initial drafts of this article were greatly appreciated.

\section{Author Note}

Address correspondence to: Kami L. Schwerdtfeger, Ph.D., Human Development and Family Studies, Oklahoma State University, 233 HES, Stillwater, Oklahoma 74078. E-MAIL: kami.schwerdtfeger@okstate.edu.

\section{Authors' Biographical Sketches}

Kami L. Schwerdtfeger is Assistant Professor of Marriage and Family Therapy at Oklahoma State University in Stillwater, Oklahoma. Her current research activities pertain to the effects of trauma on women's mental health and childbearing issues.

Briana S. Nelson Goff is Assistant Dean for Academic Affairs in the College of Human Ecology and Associate Professor in the School of Family Studies and Human Services at Kansas State University in Manhattan, Kansas. Her current research activities pertain to examining post-traumatic stress disorder and the effects of traumatic stress on couple and family systems. E-MAIL: bnelson@ksu.edu.

\section{References}

Boothroyd, R. A. \& Best, K. A. (2003). Emotional reactions to research participation and the relationship to understanding of informed consent disclosure. Social Work Research, 27, 242-251.
Briere, J. (1996). Psychometric review of the Trauma Symptom Checklist-40. In B. H. Stamm (Ed.), Measurement of stress, trauma, and adaptation. Lutherville, MD: Sidran Press. 
Carlson, E. B., Newman, E., Daniels, J. W., Armstrong, J., Roth, D., \& Loewenstein, R. (2003). Distress in response to and perceived usefulness of trauma research interviews. Journal of Trauma and Dissociation, 4, 131-142.

Gold, J. W. \& CARdena, E. (1998). Convergent validity of three posttraumatic symptom inventories among adult sexual abuse survivors. Journal of Traumatic Stress, 11, 173-180.

Gold, S. R., Milan, L. D., Mayall, A., \& Johnson, A. E. (1994). A cross-validation study of the Trauma Symptom Checklist: The role of mediating variables. Journal of Interpersonal Violence, 9, 12-26.

Griffin, M. G., Resick, P. A., Waldrop, A. E., \& Mechanic, M. B. (2003). Participation in trauma research: Is there evidence of harm? Journal of Traumatic Stress, 16, 221-227.

Kassam-Adams, N. \& Newman, E. (2002). The reactions to research participation questionnaires for children and for parents (RRPQ-C and RRPQ-P). General Hospital Psychiatry, 24, 336-342.

KIPNIS, K. (2005). Vulnerability in research subjects: a bioethical taxonomy. Ethical and Policy Issues in Research Involving Human Participants: Commissioned Papers and Staff Analysis. The Online Ethics Center for Engineering and Science. Retrieved March 15, 2007 from http://onlineethics.org/ cite-link.html.

Loveland Cook, C. A., Flick, L. H., Homan, S. M., Campbell, C., McSweeney, M., \& Gallagher, M. E. (2004). Posttraumatic stress disorder in pregnancy: Prevalence, risk factors, and treatment. Obstetrics \& Gynecology, 103, 710-717.

Newman, E., Risch, E., \& Kassam-Adams, N. (2006). Ethical issues in trauma-related research: A review. Journal of Empirical Research on Human Research Ethics, 1, 29-46.
Newman, E., Walker, E. A., \& Gefland, A. (1999). Assessing the ethical costs and benefits of trauma-focused research. General Hospital Psychiatry, 21, 187-196.

Newman, E., Willard, T., Sinclair, R., \& KaloupeK, D. (2001). Empirically supported ethical research practice: The costs and benefits of research from the participants' view. Accountability in Research, 8, 309-329.

Parslow, R. A., Jorm, A. F., O’Toole, B. I., Marshall, R. P., \& Grayson, D. A. (2000). Distress experienced by participants during an epidemiological survey of posttraumatic stress disorder. Journal of Traumatic Stress, 13, 465-471.

Schwerdtfeger, K. L. \& Nelson Goff, B. S. (2007). The intergenerational transmission of trauma: Exploring motherfetus prenatal attachment. Journal of Traumatic Stress, 20, 39-51.

Seng, J. S., Low, L. K., Sparbel, K. J. H., \& Killion, C. (2004). Abuse-related post-traumatic stress during the childbearing year. Issues and Innovations in Nursing Practice, 46, 604-613.

U.S. National Commission for the Protection of Human Subjects of Biomedical and Behavioral Research. (1979). The Belmont report: Ethical principles and guidelines for the protection of human subjects of research. Office of the Secretary, Department of Health, Education, and Welfare.

Vrana, S. \& Lauterbach, D. (1994). Prevalence of traumatic events and post-traumatic psychological symptoms in a nonclinical sample of college students. Journal of Traumatic Stress, 7, 289-302.

Walker, E. A., Newman, E., Koss, M., \& Bernstein, D. (1997). Does the study of victimization revictimize research participants? General Hospital Psychiatry, 19, 403-410. 
